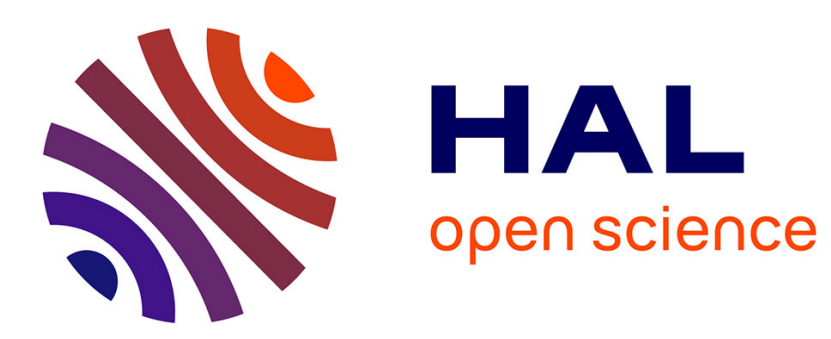

\title{
On the equiconvergence of eigenfunction expansions associated with ordinary linear differential operators
}

\author{
V. Komornik
}

\section{To cite this version:}

V. Komornik. On the equiconvergence of eigenfunction expansions associated with ordinary linear differential operators. RR-0356, INRIA. 1985. inria-00076201

\section{HAL Id: inria-00076201 \\ https://hal.inria.fr/inria-00076201}

Submitted on 24 May 2006

HAL is a multi-disciplinary open access archive for the deposit and dissemination of scientific research documents, whether they are published or not. The documents may come from teaching and research institutions in France or abroad, or from public or private research centers.
L'archive ouverte pluridisciplinaire HAL, est destinée au dépôt et à la diffusion de documents scientifiques de niveau recherche, publiés ou non, émanant des établissements d'enseignement et de recherche français ou étrangers, des laboratoires publics ou privés. 


\section{Rapports de Recherche}

$$
\mathrm{N}^{\circ} 356
$$

\section{ON THE EQUICONVERGENCE OF EIGENFUNCTION EXPANSIONS \\ ASSOCIATED WITH ORDINARY LINEAR DIFFERENTIAL OPERATORS}

Vilmos KOMORNIK

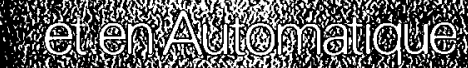

to

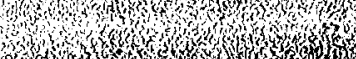

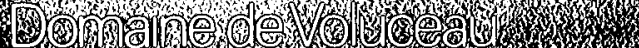
\% How

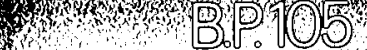


ON THE EQUICONVERGENCE OF EIGENFUNCTION EXPANSIONS ASSOCIATED WITH

ORDINARY LINEAR DIFFERENTIAL OPERATORS

\author{
VIImOS KOMORNIK \\ Eotvos Lorand University \\ Mathematical Institute \\ H-1088 Budapest \\ Muzeum Krt. 6-8 \\ HUNGARY
}

Résumé

On considère les séries de Fourier d' une fonction de carré sommable, correspondant aux systèmes orthonormaux complets constitués des fonctions propres des opérateurs différentiels linéaires ordinaires. Toutes ces séries se comportent localement de la même manière. Le résultat reste valable pour les bases de Riesz.

\title{
Abstract
}

We consider the Fourier series of a square-integrable function, corresponding to the complete orthonormal systems consisting of eigenfunctions of ordinary linear differential operators. All these series behave locally in the same way. The result remain valid for $\mathrm{Ri} e s z$ bases, too.

Chercheur invité, pour une durée de deux mois, dans le projet de MM. BENSOUSSAN/ LEMARECHAL : THEOSYS (THEOrie des SYStèmes). 
On the equiconvergence of eigenfunction expansions associated with ordinary linear differential operators

\section{Komornik}

The equiconvergence theorems are very useful in the spectral investigation of differential operators because many results known for the most special operators may be transferred by their application to more general ones. For the case of orthonormal bases consisting of eigenfunctions of second-order operators several results ave been obtained since the beginning of this century, see e.g. $[1],[2],[3],[5],[7],[8],[14],[19]$, $[22],[23]$. All these results are contained in a result of I. Joo and the author in [10]. It concerns also the nonselfadjoint case i.e. when eigenfunctions of higher order are also used and when the system is not orthonormal but only a Riesz basis. ( $O n$ the existence of such Riesz bases see [4], $[11],[20],[21]$.$) The proof was based on an efficient method$ due to V.A. II'in (see e.g. [2]) of the constant application - of some mean value formulas.

The aim of the present paper is to extend this result for differential operators of higher order. In some special cases this was already done in [15]. Our main tool will be a generalized Titchmarsh type formula derived in [12]. We note that it is not a mean value formula if the order of the differential operator is odd. In some cases a simpler expiession was tound for its' coefificients by I. Joó in [9]; these expressions are important because they make possible to obtain sharp estimates for the coeficicients. Wie shall need several results proved in the papers $[10],[17]$ and $[18]$, too. 
By and large the following result will be proved: all Riesz bases (and in particular all orthonormal bases) consisting of eigenfunctions (maybe also of higher order) of some n-order linear differential operator locally behave in the same way. Here we stress two circumstances:

- there are no assumptions on the distribution of the eigenvalues: they can be arbitrary complex numbers;

- there are no boundary conditions.

As an immediate consequence of this result we note that (for example) Carleson's theorem remains valid for "all" eigenfunction expansions.

All the preliminary results used in this paper are contained in $[10],[12],[17]$ and [18]. All the results of the papers [12] and [17] are needed. From [18] we need the case $i=0$ of Theorem 3 ; for its proof it is not necessary to apply the results of the paper [16]. From [10] we use only a result of technical character (Iemma 6). For the reader's convenience we collect in section 2 all preliminary results used in this paper. 
1. Formulation of the main result

Let $G$ be an open interval on the real line,

$n$ a natural number, $q_{S} \in H_{I O C}^{n-s}(G)$ a complex function $(s=2, \ldots, n)$ and consider the differential operator

$$
\dot{I} \cdot u=u^{(n)}+q_{2} u^{(n-2)}+q_{3} u^{(n-3)}+\cdots+q_{n} u
$$

defined on $\mathrm{H}_{\mathrm{loc}}^{\mathrm{n}}(G)$ - (Recall that, by definition, $\mathrm{H}_{\mathrm{loc}}^{\mathrm{k}}(G)$ is the set of all complex functions $v \in I_{l o c}^{2}(G)$ having distributional derivatives in $I_{l o c}^{2}(G)$ of order up to $k:$ )

As usual, a function $u \neq 0$ is called an eigenfunction of order 0 (of the operator $L$ ) with some eigênvalue $n \in \mathbb{C}$ if

$$
I u=\lambda u
$$

Furthermore, a function $u$ is called an eigenfunction of order $k$ (of the operator $I$ ) with some eigenvalue $\boldsymbol{\lambda} \in \mathbb{C}$ $(k=1,2, \ldots)$ if the function

$$
u^{*}:=\mathrm{L} u-\lambda u
$$

is an eigenfunction of order $k-1$ with the same eifenvalue $\lambda$. Let now given a system $\left(u_{r}\right)_{r=1}^{\infty}$ of eigenfunctions and denote $o_{r}$ (resp. $n_{r}$ ) the order (resp. the eigenvalue) of $u_{r}$. Assume that the following three conditions are satisfied:

(c l) $\left(u_{r}\right)$ is a Riesz basis i.e. $\left(u_{r}\right)$ is the image of an orthonormal basis under a linear topological iso- 
$-4-$

morphism of $\mathrm{I}^{2}(G)$;

(c 2) $\sup o_{r}<\infty$;

(c 3 ) if $o_{r} \geqslant 1$ then $u_{r}^{*}=u_{s}$ for some index $s$.

By (C I) there exists a unique system ( $\left.v_{r}\right)$ in $I^{2}(G)$ such that

$$
\left\langle u_{r}, v_{s}\right\rangle=\delta_{r s} \quad(\text { the kronecker symbol }) .
$$

Introduce the following notations:

$$
\begin{aligned}
& \text { (1) }\left|\nu_{r}\right|=\max \left\{|\operatorname{Im} \mu|: \mu \in \mathbb{C} \text { and } \mu^{n}=\pi_{r}\right\} \text {, } \\
& \sigma_{y}(f, x)=\sum_{\left|\nu_{r}\right|\langle\nu}\left\langle f, \nabla_{r}\right\rangle u_{r}(x) \\
& \left(\gamma>0, f \in I^{2}(G), x \in G\right), \\
& \therefore S_{y}(f, x, R)=\int_{x-R}^{x+R} \frac{\sin \gamma(y-x)}{\pi(y-x)} f(y) d y \\
& \left(y>0, f \in L^{2}(G), x \pm R \in G\right) .
\end{aligned}
$$

The aim of this paper is to prove the following result: The or e $n$. To any compact suininterval $i \mathrm{i}$ of $G$ there exists a number $R_{0}>0$ such that

$$
\lim _{\gamma \rightarrow \infty} \sup _{x \in \mathbb{K}}\left|S_{\gamma}(f, x, R)-\sigma_{\gamma}(f, x)\right|=0
$$

whenever $f \in I^{2}(G)$ and $0<R<R_{0}$. 
$\mathrm{k}$ e m a r ks.

(i) We note that $\mathrm{S} y(f, x, R)$ does not depend on the system $\left(u_{r}\right)$ :

(ii) The conditions of the theorem are very weak. The assumptions (C I) - (C 3) are practically satisfied for many Riesz bases of eifenfunctions. Wie emphasize that there ise no assumptions on the distribution of the eigenvalues $\lambda_{r}$. Several sufficient conditions are known for the existence of orthonormal bases of eigenfunctions (see e.g. [21]), and more generally, for the existence of Riesz bases of eizenfunctions (see $[4],[11],[20]$ ).

(iii) For second-order operators several equiconvergence theorems were proved from the beginning of this century, see e.g. $[1],[2],[3],[5],[7],[8],[14],[49],[22]$. These results are contained in a theorem of Joó and Komornik, proved in [10] by developing an important method of V. A. II' in [2]. This result is also a slightly stronger than the case $\mathrm{n}=2$ of the above theorem: instead of $\mathrm{q}_{2} \in \mathrm{I}_{\mathrm{Ioc}}^{2}(G)$ it was sufficient to assume that $q_{2} \in I_{I O C}^{1}(G)$.

(iv) Tiie proof of the just mentioned result of Joo and Konornik is not applicable for the general case. However, by interrating by parts we obtain the desired estirnates also in this case. On the other hand, in [12] a new method. (based on a suitable generalization of the well-known Tibcharsh formula) vias developed for the spectrai inyestifetion of n-oräer differential operators. Using this method, several results were proved, see e.g. [12]-[18] . The present paper represents a rev $\in$ vidence for the efficiency of this method. 
Some special cases of the theorem of the present paper were proved in [15].

\section{Freliminary results}

A./ We shall need the following estimate, being a consequence of some results of the papers $[17],[18]$ : putting

(2) $\left|\rho_{r}\right|=\min \left\{|\operatorname{Re} \mu|: \mu \in \mathbb{C}\right.$ and $\left.\mu^{n}=\lambda_{r}\right\}$, to any compact intervals $\mathrm{K}_{I} \subset \mathrm{G}, \mathrm{K}_{2} \subset$ int $\mathrm{K}_{1}$ there exists a positive constant $\varepsilon_{0}$ such that

$$
\text { (3) }\left\|u_{r}\right\|_{I^{\infty}\left(K_{2}\right)} e^{\left|\rho_{r}\right| \cdot \varepsilon_{0}} \leqslant \frac{1}{\varepsilon_{0}}\left\|u_{r}\right\|_{L^{2}\left(K_{I}\right)} \quad(r=1,2, \ldots)
$$

.. B./ In [12] we derived a generalization of the well-known Titchmarsh formula for n-order operators; the results cited in A./ were proved by the use of this formula. Now we need another generalization of the Titchmarsh formula.

Deriote $w_{1}, \ldots, w_{n}$ the $n$-th roots of unity and set $\mathrm{m}=\left[\frac{\mathrm{n}+1}{2}\right]$. For $0 \neq \mu \in \mathbb{C}, t>0$ and

- m $t<y<(n-m) t$ we denote by $f_{k}(\mu, t)$ the elenentary symetric polynomial of degree $m-k$ of the variajles

$$
\begin{aligned}
& \mathrm{e}^{\mu w_{1} t}, \ldots, \mathrm{e}^{\mu u_{n} t} \text { with the main coefficient }(-1)^{k} \\
& (k=m-n, \ldots, m) \text { and we put }
\end{aligned}
$$

$$
F(\mu, t, y)=\sum_{k=1+\left[\frac{-y}{t}\right]}^{m} f_{k}(\mu, t) \sum_{p=1}^{n} \frac{\omega_{p}}{n \mu^{n-I}} e^{\mu \omega_{p}(y+k t)}
$$


One can easily see that $f_{k}$ and $F$ can be continuously extended for all $\mu \in \mathbb{C}, t \geqslant 0$ and $-m t \leqslant y \leqslant(n-m) t$. Furthermore, the extended function $F$ has the following properties for any fixed $\mu \in \mathbb{C}$ and $t>0$ :

(4) $F(\mu, t, \cdot)$ is $n-2$ times continuously differentiable in $(-m t,(n-m) t)$ and

$D \frac{i}{3} F(\mu, t,-m t+0)=D_{3}^{i} F(\mu, t,(n-m) t-0)=0 \quad(i=0, \ldots, n-2)$.

(5) $\mathrm{F}(\mu, t, \cdot)$ is $\mathrm{n}$ times continuously differentiable in $(k t,(k+1) t)$ and $D_{3}^{n} F=\mu^{n} F(-m \leqslant k \leqslant n-m-1)$.

(6) $-D_{3}^{n-1} F(\mu, t,(n-m) t-0)=f_{m-n}(\mu, t)$,

$$
\begin{gathered}
D_{3}^{n-1} \cdot F(\mu, t,-k t+0)-D_{3}^{n-1} F(\mu, t,-k t-0)=f_{k}(\mu, t) \\
(m-n<k<m),
\end{gathered}
$$$$
D_{3}^{n-1} F(\mu, t,-m t+0)=f_{m}(\mu, t) \text {. }
$$

üsing these properties anc integrating by parts we obtain for any $u \in \mathrm{H}_{\mathrm{loc}}^{\mathrm{n}}(G)$ the fornula

$$
\text { (7) } \left.\left.\sum_{k=m-n}^{m} f_{k}(\mu, t) u(x+k t)+\int_{x+(m-n) t}^{x+m t} F(\mu, t, x-\tau)\left[\mu^{n} u(\tau)-u(n)\right] \tau\right)\right] d \tau=
$$

whenever $t>0, x+(m-n) t \in G$ and $x+m t \in G$.

c./ Apply the formula (7) to the eisenfunction $u_{r}$. Denoting by $\mu_{r}$ an arbitrary $n$-th root of $\lambda_{r}$, we obtain 
(8)

$$
\begin{aligned}
& \sum_{k=m-n}^{m} f_{k}\left(\mu_{r}, t\right) u_{r}(x+k t) \\
& +\int_{x+(m-n) t}^{x+m t} F\left(\mu_{r}, t, x-\tau\right)\left[\sum_{s=2}^{n} q_{s}(\tau) u_{r}^{(n-s)}(\tau)-u_{r}^{*}(\tau)\right] d \tau=0
\end{aligned}
$$

whenever $\quad t>0, x+(m-n) t \in G$ and $x+m t \in G$.

For $\mathrm{n}=2$ and $\mathrm{o}_{\mathrm{r}}=0$ this reduces to the Titchmarsh formula. For $\mathrm{n}=2$ and $o_{r} \neq 0$ it was found by Joo [6]. For $o_{r}=0, n$ arbitrary (then $u_{r}^{*} \equiv 0$ ) the formula (8) is a special case of a more general formula derived in [12]. we note that the above simple form of the coefficients $f_{k}$ (which has great importance to obtain some estimates in the sequel) was proved by Joó [9].

Wie shall frequently use two equivalent forms of the formula (8). Index the $\mathrm{n}$-th roots of $\lambda_{r}$ such that

$$
\operatorname{Re} \mu_{r, 1} \geqslant \ldots \geqslant \operatorname{Re} \mu_{r, n}
$$

and put $\mu_{r}=\mu_{r, \mathbb{B}}, \rho_{r}=\operatorname{Re} \mu_{r}, \nu_{r}=\operatorname{Im} \mu_{r}$.

These notations are in keeping with the former ones used in (1), (2), (3) and (8). Denote $g_{\mathrm{K}}\left(\mu_{r}, t\right)$ and $G\left(\mu_{r}, t, y\right)$ (resp. $h_{k}\left(\mu_{r}, t\right)$ and $\left.H\left(\mu_{r}, t, y\right)\right)$ the functions obtained from $f_{k}\left(\mu_{r}, t\right)$ and $F\left(\mu_{r}, t, y\right)$ by dividing by $e^{\left(\mu_{r, 1}+\ldots+\mu_{r, \mathrm{~m}-1}\right) t}\left(\operatorname{resp.} e^{\left(\mu_{r, 1}+\ldots+\mu_{r, m}\right) t}\right)$.

Then from (8) we obtain the following two formulas: 
$-9-$

(9)

$$
\begin{aligned}
& \sum_{n=m-n}^{m} g_{k}\left(u_{r}, t\right) u_{r}(x+k t) \\
& +\int_{x+(m-n) t}^{x+m t} G\left(\mu_{r}, t, x-\tau\right)\left[\sum_{s=2}^{n} q_{s}(\tau) u_{r}^{(n-s)}(\tau)-u_{r}^{*}(\tau)\right] d \tau=0 .
\end{aligned}
$$

(10) $\sum_{h=m-h}^{m} h_{k}\left(\mu_{r}, t\right) u_{r}(x+k t)$

$$
+\int_{x+(m-n) t}^{x+m t} H\left(\mu_{r}, t, x-\tau\right)\left[\sum_{s=2}^{n} q_{s}(\tau) u_{r}^{(n-s)}(\tau)-u_{r}^{*}(\tau)\right] d \tau=0 .
$$

D.) It follows obviously from (4) that

(11)

$$
\begin{aligned}
& D \frac{\dot{i}}{3} G\left(\mu_{r}, t,-m t+0\right)=D D_{3}^{i} G\left(\mu_{r}, t,(n-m) t-0\right)=0, \\
& D \frac{\dot{1}}{3} H\left(\mu_{r}, t,-m t+0\right)=D \frac{i}{3} H\left(\mu_{r}, t,(n-m) t-0\right)=0 \\
& (0 \leqslant i \leqslant n-2, \quad r=1,2, \ldots) .
\end{aligned}
$$

The following estimates follow directly from the definition of the coefficients in the formulas (8), (9), (10), we refer to $\sqrt{[17]}$ for some details. In all these estimates we assume that $\rho_{r} \geqslant 0$ and $t>0$.

(12) $\varepsilon_{k}\left(\mu_{r}, t\right), h_{k}\left(\mu_{r}, t\right), D_{2} g_{k}\left(\mu_{r}, t\right)$ and $D_{2} h_{k}\left(\mu_{r}, t\right)$ ter ci to 0 if $|k| \geqslant 2$ and $\left|\mu_{r} t_{.}\right| \rightarrow \infty$. 
(13) $g_{I}\left(\mu_{r}, t\right)$ and $g_{-I}\left(\mu_{r}, t\right)$ remain bounded,

$D_{2} g_{1}\left(\mu_{r}, t\right), D_{2} g_{-I}\left(\mu_{r}, t\right)$ and $g_{0}\left(\mu_{r}, t\right)-e^{\mu_{r} t}$

tend to 0 if $S_{r} t \rightarrow \infty$ and $\frac{\left|\nu_{r}\right|}{\rho_{r}} \rightarrow \infty$

(14) $h_{1}\left(\mu_{r}, t\right), h_{-1}\left(\mu_{r}, t\right), D_{2} h_{1}\left(\mu_{r}, t\right), D_{2} h_{-1}\left(\mu_{r}, t\right)^{\prime \prime}$ and

$h_{0}\left(\mu_{r}, t\right)-1$ tend to 0 if $\rho_{r} t \rightarrow \infty$ and

$\frac{\left|\nu_{r}\right|}{\rho_{r}}$ remains bounded.

(15) $g_{-I}\left(\mu_{r}, t\right), g_{1}\left(\mu_{r}, t\right)$ and $h_{\alpha I}\left(\mu_{r}, t\right)$ reinain bounded, $D_{2} g_{-1}\left(\mu_{r}, t\right), D_{2} g_{I}\left(\mu_{r}, t\right), g_{I}\left(\mu_{r}, t\right)+1$,

for $n$ odd $D_{2} h_{-1}\left(\mu_{r}, t\right)$ and $h_{0}\left(\mu_{r}, t\right)-1$,

for $n$ even $g_{-1}\left(\mu_{r}, t\right)+1$

tend to 0 if $\quad\left|\gamma_{r} t\right| \rightarrow \infty$ and $\rho_{r} t$ remains

bounded.

(16) For any real number $\nu$ the fractions

$\frac{g_{0}\left(\mu_{x}, t\right)-g_{0}(i \nu, t)}{t}$ and $\frac{h_{1}\left(\mu_{r}, t\right)-h_{1}(i \nu, t)}{t}$ 
remain bounded (uniformly in $\gamma$ ) if $\rho_{r} t$ and $\left|\mu_{r}-i \nu\right|$ remain bounded.

(17) $\frac{D \frac{i}{3} G\left(\mu_{r}, t, y\right)}{\left|\mu_{r}\right|^{i+1-n} e^{\rho_{r}(t-|y|)}}$ and $\frac{D \frac{i}{3} H\left(\mu_{r}, t, y\right)}{\left|\mu_{r}\right|^{i+1-n} e^{-S_{r}|y|}}$ are uniformly bounded $(i=0, \ldots, n-1, r=1,2, \ldots)$.

E./ It follows from Theorem 2 in [12] that for any compact subinterval $K$ of $G$ there exists a constant $C>0$ such th

$\left\|u_{r}^{\prime}\right\|_{L^{\infty}(K)} \leqslant c \cdot\left(I+\left|u_{r}\right|\right) \cdot\left\|u_{r}\right\|_{I^{\infty}(K)} \quad(r=1,2, \ldots)$.

F./ Finally we recall two important properties of the fiesz bases: the generalized Bessel inequality and the generalized Parseval identity: First, there exists a constant $C$ such that

(18) $\sum_{j=1}^{\infty}\left|\left\langle u_{r}, w\right\rangle\right|^{2} \leqslant c\|w\|_{L^{2}(G)}^{2} \quad \forall w \in I^{2}(G)$. secondily,
(19) $\langle f, w\rangle=\sum_{r=1}^{\infty}\left\langle f, v_{r}\right\rangle\left\langle u_{r}, w\right\rangle$
$\forall f, w \in L^{2}(G)$

3. Estination of the sun of squares of the eigenfunctions

In this section, under the conditions of the Theorem. we shall prove the following strong estimate: 
F r o p o s i t $i \circ n$. To any compact subinterval $K$ of $G$ there exists a positive number $\varepsilon$ such that

$\sup _{y \geqslant 1} \sum_{|\gamma-| \nu_{+} \| \leqslant 1}\left\|u_{r}\right\|_{L^{\infty}(K)}^{2} e^{\left|\rho_{r}\right| \cdot \varepsilon}<\infty \quad \cdot \square$ This result will follow from several lemnas. I e m m a 1 .

$$
\begin{aligned}
& \sup _{\nu \geqslant 1} \sum_{\substack{\left|\gamma-\nu_{r}\right| \leqslant 1 \\
\nu_{i} \geqslant A \cdot \rho_{r} \\
\rho_{r} \geqslant A}}\left(\frac{\left\|u_{r}\right\|_{I^{2}(K)}}{\rho_{r}}\right)^{2}=0(I) \quad(A \rightarrow \infty) . \\
& \text { Proof. Fix } R>0 \text { such that } \mathrm{K}_{2 \mathrm{nR}} \subset \mathrm{G} \text { where }
\end{aligned}
$$$$
K_{\delta}:=-\{x: \operatorname{dist}(x, K) \leq \delta\} \text {. For any } \nu \geqslant 1, x \in K \text {, }
$$$$
R \leqslant t \leqslant 2 R \text { and } r \in I_{\gamma}(A):=\left\{r:\left|\gamma-\gamma_{r}\right| \leqslant 1, \gamma_{r} \geqslant A \rho_{r}\right. \text {, }
$$$$
\left.. V_{r} \geqslant A\right\} \text {, by the application of the formula (9) we obtain }
$$

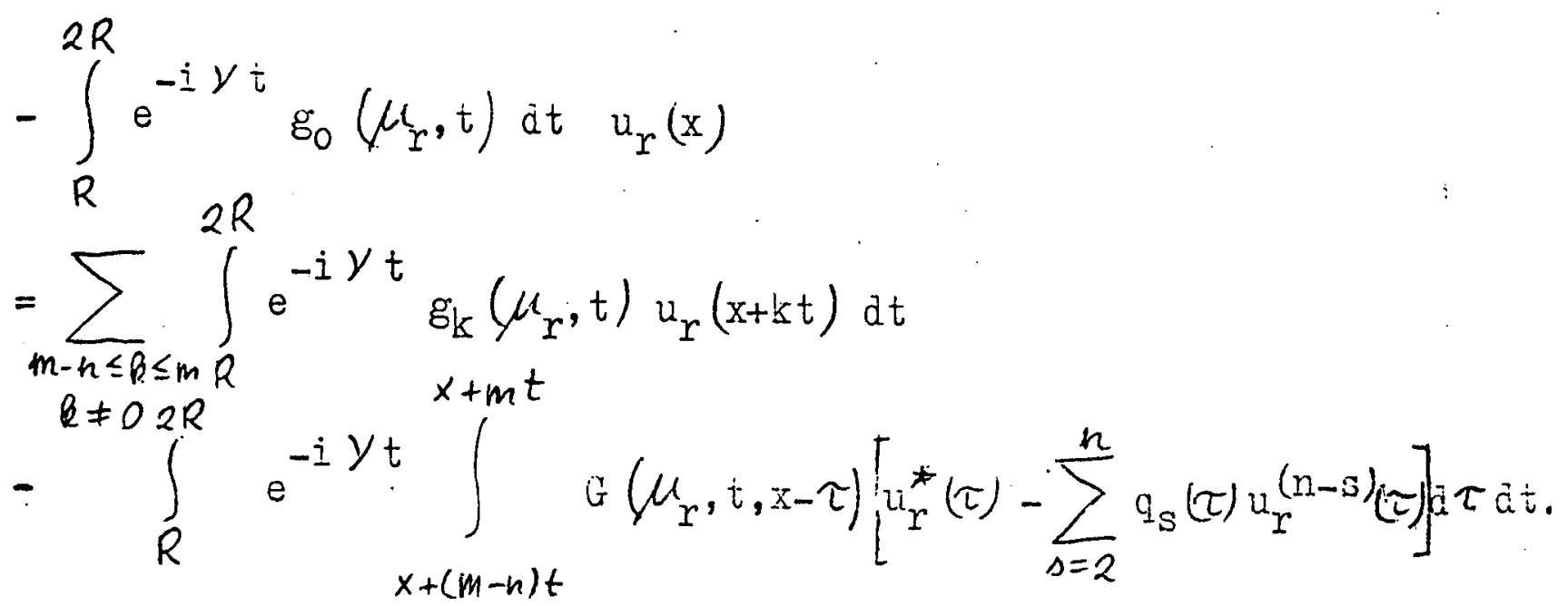

Inteyrating by parts and using (II) hence we obtain 
$-13-$

$$
\begin{aligned}
& -\int_{R}^{2 R} e^{-i \nu t} g_{0}\left(\mu_{r}, t\right) d t u_{r}(x) \\
& =\sum_{\substack{m-n \leq k \leq m \\
k \neq 0}} \varepsilon_{k}\left(\mu_{r}, 2 R\right) \int_{R}^{2 R} e^{-i \nu t} u_{r}(x+k t) d t \\
& -\sum_{m-n \leq k \leq m} \int_{R}^{2 R} D_{2} \xi_{k}\left(\mu_{r}, t\right) \int_{R}^{t} e^{-i \nu \xi} \cdot u_{r}(x+k \xi) d \xi d t \\
& +\sum_{s=2}^{n} \sum_{i=0}^{\substack{n \neq 0 \\
n-\infty}}(-1)^{i}\left(\begin{array}{c}
n-s \\
i
\end{array}\right) \int_{R}^{2 R} e^{-i \nu t} \int_{x+(m-n) t}^{x+i n t} D_{3}^{n-s-i+1} G\left(\mu_{r}, t, x-\tau\right) \\
& \text { - } \int_{x+(m-n) t}^{\tau} q_{S}^{(i)}(\xi) u_{r}(\xi) d \xi d \tau d t \\
& -\int_{R}^{2 R} e^{-i \nu t} \int_{x+(m-n) t}^{x+m t} D_{3} G\left(\mu_{r}, t, x-\tau\right) \int_{x+(m-n) t}^{\tau} u_{r}^{*}(\xi) d \xi d \tau d t .
\end{aligned}
$$

The following estimates will be uniform in $\nu, x, r, t, \tau$ when $A \rightarrow \infty$. Using the estimates (12), (13), (17), with suitably defined functions $w_{1}, w_{2}, w_{3}, w_{4} \in L^{2}(G)$ (which depend also on $V, x, t, \tau, k, s, i)$ we obtain

$$
\begin{aligned}
& (I-\overline{\bar{o}}(1))\left|\frac{u_{r}(x)}{\rho_{r}}\right| \leqslant \sum_{\substack{m-n \leq k \leq l n \\
k \neq 0}} 0(1)\left|\left\langle w_{1}, u_{r}\right\rangle\right| \\
& +\sum_{m-n \leq k \leq m} \int_{R}^{2 R} 0(I)\left|\left\langle w_{2}, u_{r}\right\rangle\right| d t \\
& +\sum_{n=2}^{n} \sum_{i=0}^{n-s} \int_{R}^{2 R} \int_{x+(m-i n) \div}^{x+m t} 0(1)\left|\left\langle w_{3}, u_{r}\right\rangle\right| d \tau d t \\
& +\int_{\dot{R}}^{2 R} \int_{x+(m-n) t}^{x+m+t} O(1)\left|\left\langle w_{4}, u_{r}^{*}\right\rangle\right| d \tau d t .
\end{aligned}
$$


Taking the square of this inequality, summarizing for $r \in I_{\gamma}(A)$ using $(18)$ and we obtain

$\sum_{r \in I_{\gamma}(A)}\left|\frac{u_{r}(x)}{\rho_{r}}\right|^{2} \leqslant \sum_{\substack{m-n \leqslant B \leqslant m \\ l R \neq 0}} 0(I)\left\|w_{I}\right\|_{I^{2}(G)}^{2}$

$+\sum_{m=n \leq R \leq m R} \int_{k \neq 0}^{2 R} O(I) \quad\left\|w_{2}\right\|_{L^{2}(G)}^{2} d t$

$+\sum_{s=2}^{n} \sum_{i=0}^{n-s} \int_{R}^{2 R} \int_{x+(m-n) t}^{x+m t} 0(I)\left\|w_{3}\right\|_{I^{2}(G)}^{2} d \tau d t$

$+\int_{R}^{2 R} \int_{x+(m-n) t}^{x+\ln t} 0(I)\left\|w_{4}\right\|_{I^{2}(G)}^{2} d \tau d t$.

Furthermore, one can easily see that

$$
\left\|w_{i}\right\|_{I^{2}(G)}^{2}=0(I), i=1,2,3,4 \text {, }
$$

therefore

$$
\sum_{r \in I_{y}(A)}\left|\frac{u_{r}(x)}{\rho_{r}}\right|^{2}=0(I) .
$$

Integrating on $K$, we obtain the required estimate. $\square$ $I \in m i a \quad 2$.

$$
\begin{aligned}
& \sup _{\nu \geqslant 1} \sum_{\substack{\left|\nu+\nu_{r}\right| \leq 1 \\
\nu_{r} \leq-A S_{r}}}\left(\frac{\left\|u_{r}\right\|_{L^{2}(K)}}{\rho_{r}}\right)^{2}=0(I) \quad(A \rightarrow \infty) . \\
& \text { P } r \circ \circ f \text {. Quite similar to that of Iemma } 1 \text {, replacing } \\
& e^{-i \gamma_{i}} \text { by } e^{i \gamma t} \cdot \square
\end{aligned}
$$


$-15-$

I em ma 3. For any fixed $A>0$ we have

$$
\sum_{\substack{\left|\nu_{i}\right|<A \cdot \rho_{i} \\ \rho_{i} \geqslant B}}\left\|u_{r}\right\|_{I^{2}(\mathrm{~K})}^{2}=O(1)(B \rightarrow \infty) .
$$

Pr OO I. Fix $R>0$ such that $\mathrm{K}_{2 n R} \subset G$. For any $x \in \mathbb{K}, R \leq t \leq 2 R, r \in I(B):=\left\{r:\left|\nu_{r}\right|<A \cdot \rho_{r}, \dot{\rho}_{r} \geqslant B\right\}$, applying now the formula (10), integrating by parts and using (11), we obtain

$$
\begin{aligned}
& -\int_{R}^{2 R} h_{0}\left(\mu_{r}, t\right) d u_{r}(x)=\sum_{\substack{m-n \leq k \leq m \\
R \neq 0}} h_{k}\left(\mu_{r}, 2 R\right) \int_{R}^{2 R} u_{r}(x+k t) d t \\
& -\sum_{\substack{m-h \leq k \leq m \\
k \neq 0}}^{2 R} \int_{R}^{2} D_{2} h_{k}\left(\mu_{r}, t\right) \int_{R}^{t_{R}^{k \neq 0}} u_{r}(x+k \xi) d \xi d t \\
& -\sum_{s=2}^{n} \sum_{i=0}^{h \neq 0}(-1)^{i}\left(\begin{array}{c}
n-s \\
i
\end{array}\right) \int_{R}^{2 R} \int_{x+(m-n) t}^{x+m t} D_{3}^{n-s-i+1} H\left(\mu_{r}, t, x-\tau\right) \\
& \cdot \int_{x+(m-n) t}^{\tau} q_{S}^{(i)}(\xi) u_{r}(\xi) d \xi d \tau d t \\
& -\int_{R}^{2 R} \int_{x+(m-n) t}^{\substack{x+(m-n) t \\
x+m t}} D_{3} H\left(\mu_{r}, t, x-\tau\right) \int_{x+(m-n) t}^{\tau} u_{r}^{*}(\xi) d \xi d \tau d t .
\end{aligned}
$$

The following estimates will be uniform in $x, r, t, \tau$ when $B \rightarrow \infty$. Using the estimates (12), (14), (17), with suitably defined functions $w_{5}, w_{6}, w_{7}, w_{8} \in I^{2}(G)$ (having 
(2) $-16-$

also the parameters $x, t, \tau, k, s, i)$ we obtain

$$
\begin{aligned}
& (1-\bar{\sigma}(1))\left|u_{r}(x)\right| \leqslant \sum_{m-n \leq b \leq m} 0(1)\left|\left\langle w_{5}, u_{r}\right\rangle\right| \\
& +\sum_{m-n \leq k \leq m} \int_{R}^{2 R} 0(1)\left|\left\langle w_{6}, u_{r}\right\rangle\right| d t \\
& B \neq 0
\end{aligned}
$$

$+\sum_{s=2}^{n} \sum_{i=0}^{n-s} \int_{R}^{2 R} \int_{x+(m-n) t}^{x+m t} 0(I)\left|\left\langle w_{7}, u_{r}\right\rangle\right| d \tau d t$

$+\int_{R}^{2 R} \int_{x+(m-h) t}^{x+m t} 0(1)\left|\left\langle w_{8}, u_{r}^{*}\right\rangle\right| d \tau d t$.

Furthermore we have

$$
\left\|w_{i}\right\|_{L^{2}(G)}^{2}=O(I) \quad, i=5,6,7,8 .
$$

and the proof can be finished by the same way as in Lemma I. $\square$ I e m n a 4 . For any fixed, B $>0$ we have

$$
\sup _{\nu \geqslant 1} \sum_{\substack{\left|\nu-\nu_{i}\right| \leq 1 \\ 0 \leqslant \rho_{r}<B \\ \nu_{r} \geqslant 0}}\left\|u_{r}\right\|_{L^{2}(K)}^{2}=\stackrel{0}{=}(I) \quad(D \rightarrow \infty) .
$$


Froof. Fix $0<R_{0}<\frac{|K|}{4}$ such that $\mathbb{K}_{4 n R_{0}} \subset G$ $(|K|$ denotes the lengtin of $K)$. Vie will show that for any fixed $0<R<R_{0}$, we have the estinate

(20) $\sum_{i \in I}\left|u_{r}(y)\right|^{2} \leqslant c(I+\overline{\overline{0}}(I)) R \sum_{i \in I}\left\|u_{r}\right\|_{L^{2}(K)}^{2}+0(I)$

$(D \rightarrow \infty)$ uniformly in $\mathcal{V} \geqslant I, y \in K$ and uniformly for any finite subset $I$ of

$$
J_{\gamma}(D):=\left\{r:\left|\gamma-\gamma_{r}\right| \leqslant I, 0 \leqslant \rho_{r}<B, \quad \gamma_{r} \geqslant D\right\}
$$

( $C$ is an absolut constant). Hence the lemma will follow easily. Indeed, integrating on $\mathrm{K}$ we obtain

$\sum_{r \in I}\left\|u_{r}\right\|_{L^{2}(K)}^{2} \leqslant c|K|(I+\overline{\bar{o}}(I)) \cdot R \sum_{r \in I}\left\|u_{r}\right\|_{I(K)}^{2}+0(I)$.

Choose at the beginning of the proof $R$, so small that

$$
C|K| R<\frac{1}{2},
$$

then, being all the terms finite by the choice of. I,

$$
\sum_{i \in I}\left\|u_{r}\right\|_{L^{2}(K)}^{2}=0(1),
$$

and, being I $C J_{y}(D)$ arbitrary, 
$-18-$

$$
\sum_{i \in \mathcal{F}_{\nu}(D)}\left\|u_{r}\right\|_{I^{2}(K)}^{2}=0(1)
$$

as stated in the lemia.

Denote $c$ the centre of $\bar{K}$. We prove (20) differently in the following three cases:

a. $\mathrm{y} \geqslant \mathrm{c}$.

b./ $\mathrm{y} \leqslant \mathrm{c}$ and $\mathrm{n}$ is even,

c./ $y \leqslant c$ and $n$ is odd.

a./ Applying the formula (10) with $\mathrm{x}=\mathrm{y}-\mathrm{t}$ we obtain

$$
\begin{aligned}
& -\int_{R}^{2 R} g_{l}\left(\mu_{r}, t\right) d t u_{r}(y) \\
& =\int_{R}^{2 R}\left(g_{0}\left(\mu_{r}, t\right)-g_{0}(i \gamma, t)\right) u_{r}(y-t) d t \\
& +\int_{R}^{2 R} g_{0}(i \gamma, t) u_{r}(y-t) d t \\
& +\sum_{\substack{m-n \leq k \leq m \\
k \neq 0,1}} g_{k}\left(\mu_{r}, 2 R\right) \int_{R}^{2 R} u_{r}(y-t+k t) d t \\
& -\sum_{m-n \leq k \leq m} \int_{R}^{2 R} D_{2} g_{k}\left(\mu_{r}, t\right) \int_{R}^{t} u_{r}(y-\xi+k \xi) d \xi d t \\
& +\sum_{s=2}^{n} \sum_{i=0}^{n \neq 0,1}(-1)^{i}\left(\begin{array}{c}
n-i \\
i
\end{array}\right) \int_{R}^{2 R} \int_{y-t+\left(r_{n}-n\right) t}^{y-t+m t} D_{3}^{n-s-i+1} G\left(\mu_{r}, t, y-t-\tau\right) \\
& \cdot \int_{-t+(m-n) t}^{\tau} q_{S}^{(i)}(\xi) u_{r}(\xi) d \xi d \tau d t
\end{aligned}
$$


$-19-$

$$
-\int_{R}^{2 R} \int_{y-t+(m-h) t}^{y-t+m t} D_{3} G\left(\mu_{r}, t, y-t-\tau\right) \int_{y-t+(m-n) t}^{\tau} u_{r}^{*}(\xi) d \xi d \tau d t .
$$

The following estimates are uniform in $\nu, y, r, t, \tau$ when $D \longrightarrow \infty$. Introducing the functions $w_{9}, \ldots, w_{13}$ $\epsilon I^{2}(G)$ (depending also on the parameters $\mathcal{\nu}, \mathrm{y}, \tau, t$, $k, s, i)$ in a suitable way, by (12), (15), (16) and (17) we have

$$
\begin{aligned}
& (R-\overline{\bar{o}}(I))\left|u_{r}(y)\right| \leqslant c R^{3 / 2}\left\|u_{r}\right\|_{L^{2}(K)}+\left|\left\langle w_{g}, u_{r}\right\rangle\right| \\
& +\sum_{\substack{m-n \leq k \leq m \\
k \neq 0,1}} 0(1)\left|\left\langle w_{10}, u_{r}\right\rangle\right|+\sum_{\substack{m-n \leq k \leq m \\
k \neq 0,1}} \int_{R}^{2 R} 0(1)\left|\left\langle w_{11}, u_{r}\right\rangle\right| d t \\
& \cdots+\sum_{s=2}^{n} \sum_{i=0}^{k \neq 0,1} \int_{R}^{2 R} \int_{y-t+(m-h) t}^{y-t+m t} 0(1)\left|\left\langle w_{12}, u_{r}\right\rangle\right| d \tau d t \\
& +\int_{R}^{2 R} \int_{y-t+(m-h) t}^{y-\frac{1}{t+m} t} O(1)\left|\left\langle w_{13}, u_{r}^{*}\right\rangle\right| d \tau d t .
\end{aligned}
$$

Taking the square of both sides, summarizing for $r \in I$, taring into cocourit that

$$
\left\|w_{i}\right\|_{L^{2}(G)}=O(1) \quad, i=9, \ldots, 13
$$

and using (18) we obtain (20). 
$-20-$

b./ Applying the formula (10) with $x=y+t$ we obtain almost the same formula as in the preceding case, with the following changes:

- $y-t$ is replaced by ' $y+t$ everywhere,

- instead of $g_{I}\left(\mu_{r}, t\right), g_{-I}\left(\mu_{r}, t\right)$ is placed on the left hand side.

After it (20) may be derived exactly by the same manner as before because $g_{-1}\left(\mu_{r}, t\right)=-1+\bar{\partial}(1)$ by (15) (this does not remain true if $\mathrm{n}$ is odd

c./ We apply now the formula (II):

$$
\begin{aligned}
& -\int_{R}^{2 R} h_{0}\left(l h_{r}, t\right) d t u_{r}(y) \\
& =\int_{R}^{2 R}\left(h_{I}\left(\mu_{r}, t\right)-h_{l}(i \nu, t)\right) u_{r}(y+t) d t \\
& +\int_{R}^{2 R} h_{I}(i y, t) u_{r}(y+t) d t \\
& +\sum_{\substack{m-n \leq \beta \leq m \\
k \neq 0,1}} h_{k}\left(\mu_{r}, 2 R\right) \int_{R}^{2 R} u_{r}(y+k t) d t \\
& -\sum_{m-n \leq b \leq m} \int_{R}^{2 R} D_{2} h_{1 k}\left(\mu_{r}, t\right) \int_{R}^{t} u_{r}(y+k \xi) d \xi d t \\
& +\sum_{s=2}^{n} \sum_{i=0}^{n \neq 0,1}(-1)^{i}\left(\begin{array}{c}
n-s \\
i
\end{array}\right) \int_{R}^{2 R} \int_{y+(m-n) t}^{y+m t} D_{3}^{n-s-i+1} H\left(\mu_{r}, t, y-\tau\right)
\end{aligned}
$$


$-21-$

$$
\int_{y+(m-n) t}^{\tau} q_{s}^{(i)}(\xi) u_{r}(\xi) d \xi d \tau d t
$$

$$
-\int_{R}^{Q R} \int_{y+(m-n) t}^{y+m t} D_{3} H\left(\mu_{r}, t, y-\tau\right) \int_{y+(m-n) t}^{\tau} u_{r}^{*}(\xi) d \xi d \tau d t .
$$

Using (12), (15), (16) and (17), with obvious notation we obtain

$$
\begin{aligned}
& (R-\bar{a}(I))\left|u_{r}(y)\right| \leqslant c R^{3 / 2}\left\|u_{r}\right\|_{I^{2}(K)}+\left|\left\langle w_{14}, u_{r}\right\rangle\right| \\
& +\sum_{\substack{m-n \leq k \leq m \\
k \neq 0,1}} 0(I)\left|\left\langle w_{15}, u_{r}\right\rangle\right|+\sum_{\substack{m-n \leq k \leq m \\
k \neq 0,1}} \int_{R}^{2 R} 0(1)\left|\left\langle w_{16}, u_{r}\right\rangle\right| d t \\
& +\sum_{s=2}^{n} \sum_{i=0}^{n-s} \int_{R}^{2 R} \int_{y+(m-n) t}^{y+m t} O(I)\left|\left\langle w_{17}, u_{r}\right\rangle\right| d \tau d t \\
& +\int_{R}^{2 R} \int_{y+(m-h) t}^{y+m t} O(I)\left|\left\langle w_{18}, u_{r}^{*}\right\rangle\right| d \tau d t .
\end{aligned}
$$

Furthermore

$$
\left\|w_{i}\right\|_{L^{2}(G)}=O(I), i=14, \ldots, 18
$$

and (20) can be obtained as in part a./. $\square$ 
$-22-$

I e mm a 5. For any fixed $B>0$ we have

$$
\sup _{y \geqslant 1} \sum_{\substack{\left|\nu_{+} y_{+}\right| \leq 1 \\ 0 \leq \rho_{+}<B \\ y_{r} \leq-0}}\left\|u_{r}\right\|_{I^{2}(K)}^{2}=0(I) \quad(D \rightarrow \infty) .
$$

Pr $0 \circ \mathrm{f}$. This is quite similar to that of Lemma 4, replacing in the formulas the term $g_{0}$ (i $\left.\gamma, t\right)$ (resp. $h_{1}(i v, t)$ ) by

$$
g_{0}(-i \nu, t) \quad\left(\begin{array}{ll}
\operatorname{resp} . & \left.h_{1}(-i \nu, t)\right) \cdot \square
\end{array}\right.
$$


I e in m a 6: . For any fixed $B, D>0$ we have

$$
\sum_{\substack{\left|\rho_{i}\right|<B \\\left|\nu_{i}\right|<D}} \mid{ }_{i}^{i} u_{r} \|_{L^{2}(K)}^{2}<\infty .
$$

P r o.o f . He will show the existence of a constant $C$. such that

(2I) $\mathrm{R}^{2} \sum_{i \in I}\left|u_{r}(y)\right|^{2} \leqslant c R^{3} \sum_{i \in I}\left\|u_{r}\right\|_{I^{2}(K)}^{2}+c$ for any $y \in K, 0<R<\frac{|K|}{2 n}$ and for any finite subset I of $-J:=\left\{r:\left|\rho_{r}\right|<B,\left|\nu_{r}\right|<D\right\}$. Indeed, then choosing $R$ such that

$$
C R|K| \leq \frac{1}{2}
$$

integrating on $\mathrm{K}$ and taking into account that $I$ ( and therefore $\left.\sum_{i \in I}\left\|u_{r}\right\|_{L^{2}(K)}^{2}\right)$ is finite, we obtain

$$
\sum_{r \in I}\left\|u_{r}\right\|_{L(2)}^{2} \leqslant \frac{2 c|\pi|}{R^{2}}
$$

ur.iformly in I ; hence the Ierma follows. 
$-24-$

Denote again c the centre of $K$. To prove (21), we distinguish three cases:

a./ $y \geqslant c$,

b./ $\mathrm{y} \leqslant \mathrm{c}$ and $\mathrm{n} \geqslant 2$,

c./ $\mathrm{y} \leqslant \mathrm{c}$ and $\mathrm{n}=\mathrm{l}$.

a./ Apply the formula (8) with $x=y-m t, 0<t<R$, then we obtain

$$
\begin{aligned}
& -\int_{0}^{R} f_{m}\left(\mu_{r}, t\right) d t u_{r}(y) \\
& =\sum_{k=m-n}^{m-1} \int_{0}^{R}\left(f_{k}\left(\mu_{r}, t\right)-f_{k}(0,0)\right) u_{r}(y-m t+k t) d t \\
& +\sum_{k=m-h}^{m-1} \cdot \int_{0}^{R} f_{k}(0,0) u_{r}(y-m t+k t) d t \\
& +\sum_{s=2}^{n} \sum_{i=0}^{n-s}(-1)^{i}\left(\begin{array}{c}
n-s \\
i
\end{array}\right) \int_{0}^{R} \int_{y-i n t}^{y} D_{j}^{n-s-i+1} F\left(\mu_{r}, t, y-\tau\right) \\
& \cdot \int_{y-n t}^{\tau} q_{S}^{(i)}(\xi) u_{r}(\xi) d \xi d \tau d t \\
& -\int_{0}^{R} \int_{y-n t}^{y} D_{j} F\left(u_{r}, t, y-\tau\right) \int_{y-n t}^{\tau} u_{r}^{*}(\xi) d \xi d \tau d t .
\end{aligned}
$$

Being $f_{k}, F$ smooth the functions 
$-25-$

$$
\frac{f_{k}\left(\mu_{r}, t\right)-f_{k}(0,0)}{t} \text { and } D_{3}^{n-s-i+1} F\left(\mu_{r}, t, y-\tau\right)
$$

are bounded for $\left|\rho_{r}\right|<B,\left|\nu_{r}\right|<D, 0<t<R$ and y-nt $<\tau<y$. Furthermore, $\left|f_{\mathrm{m}}\left(\mu_{\mathrm{r}}, \mathrm{t}\right)\right| \equiv 1$. Therefore, introducing the functions (depending on the parameters. $\mathrm{y}, \tau, \mathrm{k}, \mathrm{s}, \mathrm{i}) \quad w_{19}, w_{20}, w_{21} \in I^{2}(G)$ in a suitable way, we obtain the estimates

$$
\begin{aligned}
& R\left|u_{r}(y)\right| \leqslant c_{1} R^{3 / 2}\left\|u_{r}\right\|_{L^{2}(K)}+\sum_{k=m-n}^{m-n}\left|\left\langle w_{19}, u_{r}\right\rangle\right| \\
& +\sum_{s=2}^{n} \sum_{i=0}^{n-s} \int_{0}^{R} \int_{y-n t}^{y} c_{1}\left|\left\langle w_{20}, u_{r}\right\rangle\right| d \tau d t \\
& +\int_{0}^{R} \int_{y-n t}^{y} c_{1}\left|\left\langle w_{21}, u_{r}^{*}\right\rangle\right| d \tau d t
\end{aligned}
$$

and

$$
\left\|w_{i}\right\|_{L^{2}(G)} \leqslant c_{1} \quad, i=19,20,21
$$

for some constant $C_{1}$. Fence (21) follows by the usual way. b./ Apply now the formula (8) with $x=y+(n-m) t$, $0<t<R$, then 


$$
\begin{aligned}
& -\int_{0}^{R} f_{m-n}\left(\mu_{r}, t\right) d t u_{r}(y) \\
& =\sum_{k=m-n+1}^{m} \int_{0}^{R}\left(f_{k}\left(\mu_{r}, t\right)-f_{k}(0,0)\right) u_{r}(y+(n-m) t+k t) d t \\
& +\sum_{k=m-n+1}^{m} \int_{0}^{n} f_{k}(0,0) u_{r}(y+(n-m) t+k t) d t \\
& +\sum_{s=2}^{n} \sum_{i=0}^{n-s}(-1)^{i}\left(\begin{array}{c}
n-s \\
i
\end{array}\right) \int_{0}^{R} y+n t \\
&
\end{aligned}
$$

Hence one can proceed as in the preceedirg case because $n \geqslant 2$ implies also $\left|f_{\mathrm{m}-\mathrm{n}}\left(\mu_{\mathrm{r}}, t\right)\right| \equiv 1$.

c./ This case can be treatea quite similarly to the case b./, with a sole change: before the estimations we divide the formula written just above by $e^{\mu_{r}}$. Then

$$
\left|\frac{f_{m-n}\left(\mu_{r}, t\right)}{e^{\mu_{r} t}}\right| \equiv I
$$

and the usual procedure works. $\square$ 
$-27-$

Proof of the Proposition. It follows from Lemmas $1-6$ that

$$
\sup _{\gamma \geqslant 1} \sum_{\substack{|\nu-| \nu_{r} \| \leqslant 1 \\ \rho_{r} \geqslant 0}}\left(\left.\frac{\left\|u_{r}\right\|_{I}(K)}{1+\rho_{r}}\right|^{2}<\infty\right.
$$

If $n$ is even then the condition $\rho_{r} \geqslant 0$ is always satisfied. If $\mathrm{n}$ is odd then we have also

$$
\sup _{\nu \geqslant 1} \sum_{\substack{|\nu-| y_{r} \| \leqslant 1 \\ \rho_{r} \leqslant 0}}\left(\frac{\left\|u_{r}\right\| I^{2}(K)}{1 \div \rho_{r}}\right)^{2}<\infty
$$

by a reflection principle described in the introduction

- of the paper [17]. Therefore we have in both cases

$\sup _{\nu \geqslant 1} \sum_{|\nu-| \nu_{r} \mid \| \leqslant 1}\left(\frac{\left\|u_{r}\right\| L_{L}^{2}\left(k_{I}\right)}{1+\left|s_{r}\right|}\right)^{2}<\infty$

for any compact subinterval $K_{I}$ of $G$. Applying the estimate (2) (choose $K_{2}=K$ ) hence we obtain the proposition ( with $\varepsilon=\frac{\varepsilon_{0}}{2}$ ) for example. $\square$ 


\section{Proof of the Theorem}

The idea of the proof is the following. Putting

$$
\delta\left(y,\left|\nu_{r}\right|\right)= \begin{cases}1 & \text { if } \quad \nu>\left|v_{r}\right|, \\ \frac{1}{2} & \text { if } \quad \nu=\left|\nu_{r}\right|, \\ 0 & \text { if } \quad \nu<\left|v_{r}\right|\end{cases}
$$

and

$$
w(x+t)=\left\{\begin{array}{cl}
\frac{\sin y t}{\pi t} & \text { if }|t|<R, \\
0 & \text { otherwise },
\end{array}\right.
$$

by the application of the proposition proved in the preceeding section we will show that for any compact subset $K$ of $G$

$-(.22) \sup _{y>0} \sup _{x \in K} \sum_{r=1}^{\infty}\left|\left\langle u_{r}, w\right\rangle-\delta\left(y,\left|\nu_{r}\right|\right) u_{r}(x)\right|^{2}<\infty$ whenever $R$ is sufficiently sinall ( $w \in I^{2}(G)$ depends on the parameters $\gamma$ and $R$ ). Taking into account that

$$
\begin{aligned}
& S_{y}(f, x, \bar{K})=\langle f, w\rangle=\sum_{i=1}^{\infty}\left\langle f, v_{r}\right\rangle\left\langle u_{r}, w\right\rangle, \\
& \tilde{\sigma}_{y}(f, x)=\sum_{i=1}^{\infty}\left\langle f, v_{r}\right\rangle \delta\left(y,\left|\gamma_{r}\right|\right) u_{r}(x)-\frac{1}{2} \sum_{\left|y_{r}\right|=\gamma}\left\langle f, v_{r}\right\rangle u_{r}(x)
\end{aligned}
$$

applying the Cauchy - Schwarz inequality, (22) and the proposition 
asain, we obtain

$\sup _{y>0} \sup _{x \in K}\left|S_{y}(f, x, R)-\sigma_{\gamma}(f, x)\right| \leqslant c\|f\|_{I^{2}(G)}\left(\forall f \in I^{2}(G)\right.$.

with some constant $C$ independent of $f$. Now it suffices to show that

$\lim \sup _{\gamma \rightarrow \infty}\left|S_{\gamma}(f, x, R)-\sigma_{y}(f, x)\right|=0$ $y \rightarrow \infty \quad x^{\prime} \in K$

for any $f$ from a dense subset of $L^{2}(G)$. But this last property is satisfied for any Iinite linear combination $f$ of the eigenfunctions $u_{r}$ because then $f$ is continuously differentiable and $\sigma_{y}(f, x) \equiv f(x)$ for $\gamma$ sufficiently large, therefore one can apply a classical result of the theory of Fourier series ( $s \in e$ [25], Volume 1, p. 55). The rest of tiis section is devcted to the proof of the estimate (22). In the sequel we shall consider only the case $n>1$ because the case $n=I$ (then $I u=u^{\prime}$ ) can be easily led to the cese $n=2$ ( $\left.n u=u^{\prime \prime}\right)$. 
I e mma 7 . We have $\left|\frac{1}{R} \int_{R}^{2 R} e^{\mu t} d t\right| \rightarrow 0$ if $\mu \in \mathbb{C}$, Re $\mu \leqslant 0, R>0$ and $|\mu R| \rightarrow \infty$. F r o of . It suficices to show that

$\left|\frac{1}{R} \int_{R}^{2 R} e^{\mu t} d t\right| \leqslant e^{\operatorname{Re} \mu R} \cdot \min \left\{1, \frac{4}{|\operatorname{Im} \mu R|}\right\}$

For this, first we note that obviously

$$
\left|\frac{1}{R} \int_{R}^{2 R} e^{\mu t} d t\right| \leqslant e^{R e \mu R} .
$$

On the other hand, applying the theorem of Bonnet, there exist $R \leqslant R_{1}, R_{2} \leqslant 2 R \quad$ such that

$\left|\frac{1}{R} \int_{R}^{2 R} e^{\mu t} d t\right| \leqslant\left|\frac{1}{R} \int_{R}^{2 R} e^{R e \mu t} \cos \operatorname{Im} \mu t d t\right|$

$+\left|\frac{I}{\Omega} \int_{R}^{2 R} e^{\bar{R} e \mu t} \sin \operatorname{Im} \mu t d t\right|=\left|\frac{1}{\Omega} e^{R e, \mu R} \int_{R}^{R_{1}} \cos \operatorname{Im} \mu t d t\right|$ $+\left|\frac{I}{\pi} e^{R e \mu R} \int_{R}^{R_{1}} \sin \operatorname{Im} \mu t \quad d t\right| \leqslant \frac{4}{\operatorname{Im} \mu R \mid} e^{R e \mu R}$

I e m a a 8 . To any conpact intervals $\bar{K}_{1} \subset G, K \subset$ int $\ddot{h}_{1}$ 㚿宛e exists $R_{0}>0$ such that for any fixed $0<R<R_{0}$ $\sup _{x \in \mathbb{R}} \int_{0}^{R}\left|\frac{u_{r}(x-t)+u_{r}(x+t)-2 u_{r}(x) \operatorname{ch} \mu_{r} t}{t}\right| d t \leqslant$ 
$\measuredangle \quad c \frac{\ln \left|u_{r}\right|}{\left|u_{r}\right|}\left(\left\|u_{r}\right\|_{L^{\infty}\left(K_{1}\right)}+\left\|u_{r}^{*}\right\|_{I^{\infty}\left(K_{I}\right)}\right)$

whenever $\mu_{\mathrm{r}} \mid$ is sufficiently large. $\square$

Pro of . We shall use the notations of section 2 . We shall assume that $\rho_{r} \geqslant 0$. The case $S_{r}<0$ hence can be obtained by the reflection principle mentioned at the end of section 3 . Putting

$$
\begin{aligned}
v_{r}(y)=u_{r}(y)+\int_{x}^{y} \sum_{p=1}^{n} \frac{\omega_{p}}{n \mu_{r}^{n-1}} e^{\mu_{r} \omega_{p}(y-\tau)} \\
\quad \cdot\left(\sum_{s=2}^{n} q_{s}(\tau) u_{r}^{(n-s)}(\tau)-u_{r}^{*}(\tau)\right) d \tau
\end{aligned}
$$

one can reacily verify that $\nabla_{r} \in \mathrm{H}_{l o c}^{\mathrm{n}}(G)$ and $\nabla_{r}^{(n)}=\mu_{r}^{\mathrm{n}} \nabla_{r}$. Consequentiy $v_{r}$ is a linear combination of the functions

$e^{\mu_{r} \omega_{l}(y-x)}, \ldots, e^{\mu_{r} \omega_{n}(y-x)}$ 
By (3) we can fix $R_{0}>0$ such that $K_{4 n R_{0}} \subset K_{I}$ and

(23) $\left\|u_{r}\right\|_{L^{\infty}\left(K_{4 n R_{0}}\right)} \cdot e^{2 \rho_{r} R_{0}} \leqslant c\left\|u_{r}\right\|_{L^{\infty}\left(K_{I}\right)}(r=1,2, \ldots)$.

Wie shall distinguish two cases:

a./ $n$ is odd and $n>1$ i.e. $n=2 m-1, m \geqslant 2$.

b./ $n$ is even i.e. $n=2 m, m \geqslant 1$.

a./ For any $\mathrm{x} \in \mathrm{K}, 0<\mathrm{S}<2 \mathrm{R}_{0}$ and $0<t<\mathrm{S}$ the determinant

$$
\mid \begin{array}{cccc}
v_{r}(x-m s) & \cdots & e^{-m \mu_{r, p} s} & \cdots \\
\vdots & \cdots & \vdots & \\
v_{r}(x-2 s) & e^{-2 \mu_{r, p} s} & \cdots \\
\left(v_{r}(x-t)+v_{r}(x+t)-2 v_{r}(x) c h \mu_{r} t\right) & \cdots\left(2 c h \mu_{r, p} t\right. & \left.-2 c h \mu_{r} t\right) \cdots \\
v_{r}(x+2 S) & \cdots & e^{2 \mu_{r, p} s} & \cdots \\
\vdots & & \vdots & \\
v_{r}(x+(m+1) s) & \cdots & e^{(m+1) \mu_{r, p} s} & \cdots
\end{array}
$$

$(p=1, \ldots, n)$ vanishes. Expanding it according to the first colum, rith obrious notation we obtain the formia

$a\left(\mu_{r}, s\right)\left(u_{r}(x-t)+u_{r}(x+t)-2 u_{r}(x) \operatorname{ch} \mu_{r} t\right)$

$=\sum_{-m \leqslant \dot{k} \leqslant m+1} d_{k}\left(\mu_{r}, S, t\right) u_{r}(x+k S)$

$|\hat{k}| \geq 2$ 
$-33-$

$$
+\int_{x-m s}^{x+(m+1) s} D\left(\mu_{r}, s, t, x-\tau\right)\left(\sum_{s=2}^{n} q_{s}(\tau) u_{r}^{(n-s)}(\tau)-u_{r}^{*}(\tau)\right) d \tau .
$$

One can see easily (ci. (4)) that

$D j D\left(\mu_{r}, S, t,-(m+1) s\right)=D j D\left(\mu_{r}, S, t, m S\right)=0, j=0, \ldots, n-2$.

Therefore the above formula implies

$$
\begin{aligned}
& d\left(\mu_{r}, s\right)\left(u_{r}(x-t)+u_{r}(x+t)-2 u_{r}(x) \operatorname{ch} \mu_{r} t\right) \\
& =\sum_{-m \leqslant k \leqslant m+1} d_{k}\left(\mu_{r}, s, t\right) u_{r}(x+k S) \\
& \div \sum_{s=2}^{n} \sum_{i=0}^{n-s}(-1)\left(\begin{array}{c}
n-p) \\
i
\end{array}\right) \int_{x-m s}^{x+(m+1) s} D_{4}^{n-s-i} D\left(\mu_{r}, s, t, x-\tau\right) \quad q_{s}^{(i)}(\tau) u_{r}(\tau) d \tau \\
& \int_{x-m s}^{x+(m ; n) s} D\left(\mu \varepsilon_{r}, S, t, x-\tau\right) u_{r}^{*}(\tau) d \tau .
\end{aligned}
$$

Fitting

$$
Q\left(\mu_{r}, s\right)=e^{\left((m+1) \mu_{r, I}+\ldots+2 \mu_{r, m}-2 \mu_{r, m+1}-\cdots-m \mu_{r, n}\right) \cdot s},
$$

by the method of the paper $[17]$ we obtain the estimates 
$-34-$

$$
\begin{gathered}
\left|d_{k}\left(\mu_{r}, s, t\right)\right| \leqslant c_{1}\left|0,\left(\mu_{r}, s\right)\right| \cdot\left|\mu_{r} t\right| \cdot|| e^{-\mu_{r, m-1} s}|+| e^{\left(\mu_{r, m}+\mu_{r, m+1}\right) s} \\
\left|D_{4}^{j} D\left(\mu_{r}, s, t, x-\tau\right)\right| \leqslant c_{1}\left|Q\left(\mu_{r}, s\right)\right| \cdot\left|\mu_{r}\right|^{j+1-n} \cdot \\
\cdot \min \left\{1,\left|\mu_{r} t\right|\right\} \cdot e^{\rho_{r} s} ;
\end{gathered}
$$

furthermore, being $n$ odd there exists a constant $\alpha>0$ with

$\operatorname{Re} \mu_{r, \mathbb{m}-1} \geqslant \alpha \cdot\left|\mu_{r}\right|$ and $\operatorname{Re} \mu_{r, \mathbb{m}+1} \leqslant-\alpha\left|\mu_{r}\right|, \forall r$.

(In the a jove estimates $c_{1}$ denotes an absolute constant.)

Using these estimates from the formula we obtain

$$
\begin{aligned}
& \left|\frac{a\left(\mu_{r}, s\right)}{a_{a}\left(\mu_{r}, s\right)}\right| \cdot\left|\frac{u_{r}\left(x-t^{\prime}+u_{r}(x+t)-2 u_{r}(x) \operatorname{ch} \mu_{r} t\right.}{t}\right| \\
& \leqslant c_{2} \psi_{r} \mid e^{-\alpha\left|\psi_{r}\right|} \cdot\left\|u_{r}\right\|_{L^{\infty}\left(K_{2 n S}\right)} e^{\rho_{r} S} \\
& +\sum_{s=2}^{n} \sum_{i=0}^{n-s} c_{2}\left|\mu_{r}\right|^{2-s-i} \cdot \min \left\{\left|\mu_{r} t\right|^{-1}, 1\right\} \cdot\left\|u_{r}\right\|_{I^{\alpha}} \alpha_{\left(k_{2 n S}\right)} e^{\rho_{r} S} \\
& +c_{2}\left|u_{r}\right|^{2-n} \min \left\{\left|\mu_{r} t\right|^{-1}, 1\right\}\left\|u_{r}^{*}\right\| I_{I^{\infty}\left(K_{2 n s}\right)} e^{\rho_{r^{S}}}
\end{aligned}
$$

with another absolute constant $\mathrm{C}_{2}$. 
Let us now iix $\quad 0<R<R_{0}$ arbitrarily. If $\left|\mu_{r}\right|>\frac{1}{R}$ then

$$
\begin{aligned}
\int_{0}^{R} \min \left\{\left|\mu_{r} t\right|^{-1}, I\right\} d t & \leq \int_{0}^{\left|\mu_{r}\right|^{-1}} 1 d t+\int_{\left|\mu_{N}\right|^{-1}}^{R}\left|\mu_{r}\right|^{-1} d t \\
& =\left|\mu_{r}\right|^{-1}\left(1+\ln R+\ln \left|\mu_{r}\right|\right),
\end{aligned}
$$

therefore if $R<S<2 R$ and $\left|\mu_{r}\right|>\max \left\{1, \frac{1}{R}\right\}$ then

$$
\begin{aligned}
& \left|\frac{a\left(\mu_{r}, s\right)}{a\left(\mu_{r}, s\right) \mid}\right| \int_{0}^{R}\left|\frac{u_{r}(x-t)+u_{r}(x+t)-2 u_{r}(x) \cdot \operatorname{ch} \mu_{r} t}{t}\right| d t \\
& \leqslant c_{3}: \frac{1+\ln \left|\mu_{r} R\right|}{\left|\mu_{r}\right|}\left(\left\|u_{r}\right\|_{L^{\infty}\left(K_{4 n R}\right)}+\left\|u_{r}^{*}\right\|_{I^{\infty}\left(K_{4 n R}\right)}\right) e^{2 \rho_{r} R}
\end{aligned}
$$

If $\left|\mu_{\mathrm{r}}\right|$ is sufficiently large then by Lenma 7 we have

$$
\int_{R}^{2 R}\left|\frac{a\left(\mu_{r}, S\right)}{Q\left(\mu_{r}, S\right)}\right| \cdot d S>\frac{R}{2}
$$

whence

$$
\begin{aligned}
& \int_{0}^{R}\left|\frac{u_{r}(x-t)+u_{r}(x+t)-2 u_{r}(x) \operatorname{ch} \mu_{r} t}{t}\right| d t \\
& \leqslant c_{4} \frac{\ln \left|u_{r}\right|}{\mu_{r} \mid}\left(\left\|u_{r}\right\|_{L^{\infty}\left(K_{4 n R}\right)}+\left\|u_{r}^{*}\right\| L_{L^{\infty}\left(K_{4 n R}\right)}\right) e^{2 \rho_{r}^{R}}
\end{aligned}
$$


with some constant $C_{4}$ depending only on $R$. Taking into account (23) and the condition ( C 3) hence the Lemma foliows.

b./ For any $x \in \mathrm{h}, 0<s<2 \mathrm{R}_{0}$ and $0<t<s$ the determinant

$$
\begin{aligned}
& \mathrm{v}_{r}(\mathrm{x}) \quad \cdots \quad 1 \\
& \left(v_{r}(x-t)+v_{r}(x+t)-2 v_{r}(x) \operatorname{ch} \mu_{r} t\right) \cdots\left(2 \operatorname{ch} \mu_{r, p} t-2 \operatorname{ch} \mu_{r} t\right) \cdots \\
& \left(v_{r}(x-2 s)+v_{r}(x+2 s)\right) \cdot \cdots\left(2 \operatorname{ch} 2 \mu_{r, p} s\right) \\
& \left(v_{r}(x-m s)+v_{r}(x+m s)\right) \\
& \cdots\left(2 \operatorname{chm} \mu_{r, p} s\right) \quad \ldots \\
& (p=1, \ldots, m) \text { vanishes. Erpanding it according to the first } \\
& \text { column, with obvious notation we obtain the formula } \\
& d\left(\mu_{r}, s\right) \quad\left(u_{r}(x-t)+u_{r}(x+t)-2 u_{r}(x) \operatorname{ch} \mu_{r} t\right) \\
& =\sum_{\substack{0 \leq k \leq m \\
k \neq 1}} d_{k}\left(u_{r}, s, t\right)\left(u_{r}(x+k s)+u_{r}(x-k S)\right) \\
& +\int_{x-m s}^{x+m s} D\left(\mu_{r}, s, t, x-\tau\right)\left(\sum_{s=2}^{n} q_{s}(\tau) u_{r}^{(n-s)}(\tau)-u_{r}^{*}(\tau)\right) d \tau \text {. }
\end{aligned}
$$


- 37 -

One can see easily that

$D_{4}^{j} D\left(\mu_{r}, S, t, \pm m s\right)=0, j=0, \ldots, n-2$,

therefore

$$
\begin{aligned}
& d\left(\mu_{r}, s\right)\left(u_{r}(x-t)+u_{r}(x+t)-2 u_{r}(x) c h \mu_{r} t\right) \\
& =\sum_{\substack{0 \leq k \leq m \\
k \neq 1}} d_{k}\left(\mu_{r}, s, t\right)\left(u_{r}(x+k s)+u_{r}(x-k s)\right) \\
& +\sum_{s=2}^{n} \sum_{\substack{i=0 \\
x+m s}}^{x+m s}\left(\begin{array}{c}
i(n-s) \\
i
\end{array}\right) \int_{x-m s} D_{4}^{n-s-i} D\left(u_{r}, s, t, x-\tau\right) q_{s}^{(i)}(\tau)^{\prime} u_{r}(\tau) d \tau \\
& -\int_{x-m s} D\left(\mu_{r}, s, t, x-\tau\right) u_{r}^{*}(\tau) d \tau
\end{aligned}
$$

Putting

$$
Q\left(\mu_{r}, \mathrm{~s}\right)=e^{\left(\mathrm{m} \mu_{r, 1}+\ldots+2 \mu_{r, m-1}\right) s}
$$

we have the estimates

$$
\begin{aligned}
& \left|d_{k}\left(\mu_{r}, s, t\right)\right| \leq c_{I}\left|Q\left(\mu_{r}, s\right)\right| \cdot\left|\mu_{r} t\right| \cdot\left|e^{\left(2 \mu_{r, m}-\mu_{r, m-1}\right) s}\right| \\
& \text { if } m \geqslant 2, \\
& \left|D_{4}^{j} D\left(\mu_{r}, s, t, x-\tau\right)\right| \leqslant c_{I}\left|Q\left(\mu_{r}, s\right)\right| \cdot\left|\mu_{r}\right|^{j+1-n} \cdot \min \left\{1,\left|\mu_{r} t\right|\right\} \cdot e^{\rho_{r} s}
\end{aligned}
$$


$-38-$

Furthermore,

$$
d_{0}\left(\mu_{r}, s, t\right) \equiv 0 \text { if } \quad m=1
$$

ana there exists a constant $\alpha>0$ such that

$$
\operatorname{Re} \mu_{r, \text { Il -1 }} \geqslant \alpha \cdot\left|\mu_{r}\right| .(r=1,2, \ldots)
$$

if $m \geqslant 2$. Therefore, fixing. $0<\mathrm{R}<\mathrm{R}_{0}$. arbitrarily, hence we obtain

$$
\begin{aligned}
& \left|\frac{d\left(\mu_{r}, S\right)}{Q\left(\mu_{r}, S\right)}\right| \int_{0}^{R}\left|\frac{u_{r}(x-t)+u_{r}(x+t)-2 u_{r}(x) \operatorname{ch} \mu_{r} t}{t}\right| d t \\
& \leqslant c_{2} \cdot \frac{I+\ln \left|\mu_{r}^{R}\right|}{\left|\mu_{r}\right|}\left(\left\|u_{r}\right\|_{I^{\infty}\left(K_{4 n R}\right)}+\left\|u_{r}^{*}\right\|_{L^{\infty}\left(K_{4 n R}\right)}\right) e^{2 s_{r}^{R}}
\end{aligned}
$$

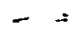

if $R<s<2 R$ and $\left|\mu_{r}\right|>\max \left\{1, \frac{1}{R}\right\}$ and the proof can be finished as in part a./.

I em ma 9 . To any $R>0$ there exists a constant c > 0 such that

$$
\left|\frac{2}{\pi} \int_{0}^{R} \frac{\sin \gamma_{t} \operatorname{cin} \mu_{r} t}{t} a t-\delta\left(\nu,\left|y_{r}\right|\right)\right| \leqslant \frac{c e^{\left|\rho_{r}\right| R}}{2+|\gamma-| y_{r}||}
$$

for all $\quad y>0$ and $r=1,2, \ldots$. 
Proof. See in $[10]$ : v

Let us now prove (22). Given a compact interval $\mathrm{K} \subset \mathrm{G}$ arbitrarily, fix another compact interval $K_{I} \subset G$ such that $K \subset$ int $K_{I}$, anỏ then a positive number $R_{I}>0$ such that

$0<R_{1}<R_{0} \quad\left(R_{0} \quad\right.$ is defined as in Lemma 8$)$, $\mathrm{K}_{4 \mathrm{nR}} \subset \mathrm{K}_{1}$,

(24) $\sup _{y \geqslant 1} \sum_{|y-| y_{r}|| \leqslant 1}\left(\left\|u_{r}\right\|_{L^{\infty}\left(I_{1}\right)} e^{\left.\left|\beta_{r}\right| R_{I}\right)^{2}}<\infty\right.$

This choice is possible by the proposition of section 3 . Fix $0<\mathrm{R}<\mathrm{R}_{1} \quad$ arbitrarily and fix a constant $A=A(R)>2$ such that the assertion of Lemma 8 hold true whenever $\left|\mu_{Y}\right|>A$. In the sequel $C$ denotes diverse constents independent of $\quad y \geqslant 1, x \in K$ and $r=1,2, \ldots$.

Consider first the case when $\left|\mu_{r}\right|>$ A. Applying Lenmas 8 and 9 we have

$$
\begin{aligned}
& \left|\left\langle u_{r}, m\right\rangle-\delta\left(y,\left|y_{r}\right|\right) u_{r}(x)\right| \\
& =\left|\int_{0}^{R} \frac{\sin y t}{\pi t}\left(u_{r}(x-t)+u_{r}(x+t)\right) d t-\delta\left(y,\left|y_{r}\right|\right) u_{r}(x)\right|
\end{aligned}
$$


$-40-$

$$
\begin{aligned}
& \leqslant\left|\int_{0}^{R} \frac{\sin \nu t}{\pi t}\left(u_{r}(x-t)+u_{r}(x+t) \cdot-2 u_{r}(x) \operatorname{ch} \mu_{r} t\right) d t\right| \\
& +\left|\frac{2}{\pi} \int_{0}^{R} \frac{\sin \gamma_{t} \operatorname{ch} \mu_{r} t}{t} d t-\delta\left(\dot{\gamma},\left|\nu_{r}\right|\right)\right| \cdot\left|u_{r}(x)\right| \\
& \leqslant \quad c\left(\frac{\ln \left|\mu_{r}\right|}{\left|\mu_{r}\right|}+\left(2+|y-| y_{r} \mid\right)^{-1}\right)\left(\left\|u_{r}\right\|_{I^{\infty}\left(K_{I}\right)}+\left\|u_{r}^{*}\right\|_{L^{\infty}\left(K_{I}\right)}\right)^{\mid s_{r} \mathbb{R} .}
\end{aligned}
$$

for all $y \geqslant I$ and $x \in K$. Using (24) and (C3) $_{\text {with any fixed }}^{\text {with }}$ $0<\varepsilon<1$, hence we obtain

$$
\begin{aligned}
& \sum_{\left.\left|u_{r}\right|\right\rangle A}\left|\left\langle u_{r}, w\right\rangle-\delta\left(\nu,\left|\nu_{r}\right|\right) u_{r}(x)\right|^{2} \\
& \leqslant c \sum_{\left|\mu_{n}\right|>A}\left(\left(1+\left|\nu_{r}\right|\right)^{-2+\varepsilon}+\left(2+|\gamma-| \nu_{r} \mid\right)^{-2}\right) \\
& \text { - }\left(\left\|u_{r}\right\|_{L^{\infty}\left(K_{I}\right)}+\left\|u_{r}^{*}\right\|_{\left.L q_{K_{I}}\right)}\right)^{2} e^{2 R_{r} \mid R_{I}} \\
& \leqslant c \sum_{i=1}^{\infty}\left(i^{-2+\varepsilon}+(I+|\gamma-i|)^{-2}\right) \sum_{i-1 \leqslant\left|\nu_{r}\right| k_{i}}\left(\left\|u_{r}\right\|_{I^{\infty}\left(K_{I}\right)} e^{\left|\rho_{r}\right| R_{I}}\right)^{2} . \\
& \leqslant c \sum_{i=1}^{\infty}\left(i^{-2+\varepsilon}+(1+|\gamma-i|)^{-2}\right) \leqslant c
\end{aligned}
$$

i.e. 
- 41 -

(25) $\sum_{u_{r} \mid>A}\left|\left\langle u_{r}, w\right\rangle-\delta\left(\gamma,\left|v_{r}\right|\right) u_{r}(x)\right|^{2} \leqslant c$.

Consider now the case when $\left|\mu_{r}\right| \leqslant A$. For any $\nu \geqslant 1$ and $x \in K$, integrating by parts and taking into account that the improper integral $\int_{0}^{\infty} \frac{\sin x}{x} d x$ is convergent, we obtain

$$
\begin{aligned}
& \left|\left\langle u_{r}, w\right\rangle\right|=\mid \int_{0}^{R} \frac{\sin \gamma t}{\pi t} d t\left(u_{r}(x-R)+u_{r}(x+R)\right) \\
& +\int_{0}^{R} \frac{\sin \gamma \xi}{\pi \xi} d \xi\left(u_{r}^{\prime}(x-t)-u_{r}^{\prime}(x+t)\right) d t \mid \\
& \leq i\left(\left\|u_{r}\right\|_{I^{\infty}\left(K_{I}\right)}+\left\|u_{r}^{\prime}\right\|_{L^{\infty}\left(K_{I}\right)}\right)
\end{aligned}
$$

But $\left|u_{r}\right|$ is bounded, therefore by the result mentioned in section 2 , part $\mathbb{E} . /$ hence we can conclude that

$$
\left|\left\langle u_{r}, w\right\rangle\right| \leqslant c\left\|u_{r}\right\|_{I^{\infty}\left(K_{I}\right)}
$$

and

$$
\left.\left|\left\langle u_{r}, w\right\rangle-\delta\left(y,\left|\gamma_{r}^{\prime}\right|\right) u_{r}(x)\right| \leq c\left\|u_{r}\right\|_{I} q_{\left(k_{I}\right.}\right)
$$


Using (24) aģain hence we obtain

(26) $\sum_{\left|\mu_{r}\right| \leq A}\left|\left\langle u_{r}, w\right\rangle-\delta\left(\gamma,\left|\nu_{r}\right|\right) u_{r}(x)\right|^{2} \leqslant c$.

(25) and (26) imply (22) and the proof of the Theorem is finished. $\square$

$\mathrm{R}$ e $\mathrm{m}$ a $\mathrm{rk}$. iie note that in the proof of the Proposition

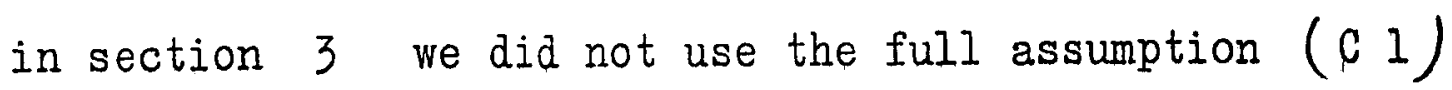
but only its consequence (18). Thus our result remains valid for all (not necessarily complete) orthonormal systems consisting of eigeniunctions of order $O$ (for example). o p e n problems.

1. It would be interesIting to know whether the assumption -.. (C 3) is necessary for the validity of the Proposition. 2. From the viewpoint of applications the Theorem proved in this paper seems to be very general and satisfactory. However, from a pure mathematical viewpoint it would be useful to enlighten whether the result remains true for the more general differential operator

$I u=u^{(n)}+q_{I} u^{(n-1)}+\ldots+q_{n} u \quad, q_{s} \in I_{I o c}^{I}(G), s=1, \ldots, ?$ 
$R$ e $f$ e $r$ e n c e s

[1] $\dot{H}$. Haar, Reichenentwicklungen nach Legendreschen Folynomen. Math. Annalen, 78 (1917) , 121-136.

[2] В.А.Ильин, Необходияые ил достаточные условия базисности и равносходиности с тригонометрическия рядом спектральних разложений 1-2. Диференцильные уравнения, 16 (1980), 771-794, 980-1009.

[3] В.А.Ильин, И.Ио, точная оценка разности частичных сумм раэлоленй, отвечающих двуя пронзвольным неотридательньа самосопрлхённим распирениям двух операторов типа Пттурка Лиувиля для абсолютной непрерывной функции. Диференциальные уравнения, 15 (1979) , 1175-1193.

[4] Н. И. Ионкин, Решение одной краевой задачи теории теплопроводности с неклассически краевњ устовием. Диференциальные уравнения, 13 (1977) , 293-304.

[5] П.А.Исматуллаев, И.Й, Теоремы о равносходимости. Acta liath. Acad. Sci. Hungar. (to appear).

[6] I. Joo, Upper estimates for the eigenfunctions of the Schrödinger operator. Acta Sci. liath., 44 (1982), 87-93.

[7] И. По, теорема типа равносходимости. ДАН $\mathrm{Y}_{3} \mathrm{CCP}, 4$ (1983), 6-8.

[8] I. Joó, On some spectral properties of the Schrödinger operator. Annales Univ. Sci. Budapest, Sect. Kiath. (to appear).

[9] I. Joó, Remarks to a paper of V. Konornik. Acta Sci. Wiath. (to anear).

[10] I. Joo - V. Komornik, on the equiconvergence of expansions by Riesz bases formed by eirenfunctions of the Schrödinger operator. Hcta Sci. hiatin. (to appear). 
[11] Г.М. Кесельман, о безусловной сходимости разложений по собственным функциям некоторых дйореренциальных операторов. Изв. вузов СССР, Натематпка, № 2 (1964), 82-93.

[12] $V$. Konornik, Upper estimates for the eigenfunctions of higher order of a linear differential operator. Ácta Sci. iivath., 45 (1983), 261-271.

[13] V. Kowornik, on the distribution of the eigenvalues of an orthonormal system consisting of eigenfunctions of higher order of a linear differential operator. Acta îth. Acad. Sci. Hungar.,42(1983), 171-175.

[14] V. Konornik, An equiconvergence theorem for the Schrödinger operator. Acta liath. Acad. Sci. Hungar. (to appear).

[15] V. Komornik, on the equiconvergence of expansions by Riesz bases formed by eigenfunctions of a 2n-order linear differential operator. Acta Hath. Acad. Sci. Hungar. (to appear,

[16] V. Komornik, Some new estimates for the eigerifunctions of higher order of a linear differential operator. Acta liath. Acad. Sci. Hungar. (to appear) .

[17]. V. Konornik, Local upper estimates for the eigenfunctions oi a linear differential operator. Acta Sci. Math. (to appear).

[18] V. Konornit, Lower estimates for the eigenfunctions of a linear differential operator. Acta ilath. Ácad. Sci. Hungar. (to appear).

[19] Б. М. Левитан, Разловение по собственным функция. Москва, 1950.

$[20]$ В. П. Іихайлов, 0 базисах Рисса в $L^{2}(0,1)$. IAH CCCP, 144,5 (1968), 981-984.

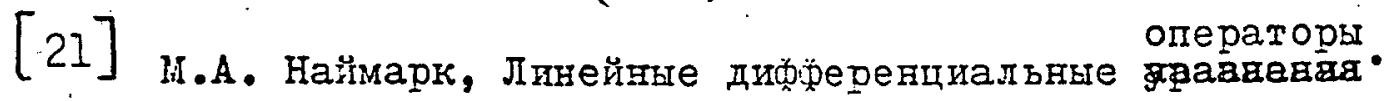
Москва, 1969. 
[22] V. A. Stekloff, Sur les expressions asymptotiques de certaines fonctions définies par des équations differentielles linéaires du deuxiene ordre, et leurs applications au probleme du développenent d'une fonction arbitraire en series procédant suivant les àites fonctions.

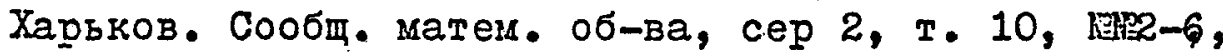
1907-1909, c. 96-199.

[23] E. C. Witchmarsh, Rigenfunction expansions associated with second-order differential equations. Oxford, Clarendon Press, 1946.

[24] A. Zygmund, Trigonometric series. Cambridge University Press, 1959.

EÖTVÖS LORAISD UNIVERSITI

MATHU⿴囗十⺝TICAL INSTITŨT

H - 1088 BUDHPEST, WUZHUi LRT. 6-8.

$\ldots$.., HUNGAIY 
